services are audited and most are managed from within a psychology department.

This book describes the theoretical background of psychodynamic psychotherapy, illustrated by case examples showing work with children, adolescents, adults and carers. It gives a clear flavour of the clinical work of the Tavistock Clinic Learning Disability Service in London, which has been built on what appears to be a niche interest within the psychotherapeutic field. It also includes an attempt at using audit to quantify the psychological gains made by clients and gives brief details of a research project that will measure outcome in a more structured way.

Service users who have been helped through psychodynamic psychotherapy are vividly portrayed, as are the dilemmas and empathy of their therapists. It is not all roses: powerful, unpleasant feelings are given expression as the therapists try to understand and the service users struggle to make sense of years of pain, loss and lack of self-worth.

It would have been interesting to be told more about the duration of treatment and why it was stopped. Several treatment contracts appear to have ended after a year, which by any standards is a short duration for this type of intervention. Occasionally I wondered what was really being said in the room, although the extracts from the sessions clarified some of the confusion about what belonged to the therapist and what to the client.

Another unaddressed issue is why clients were referred. It would have been helpful if a profile had emerged of clients who might do better with psychotherapy at the Tavistock rather than locally. In addition, data on which services tend to refer more often could help to identify service gaps within and outside of London and to indicate whether successful referral depends on a 'postcode lottery'.

Finally, information on what the Tavistock Learning Disability Service currently offers and whether funding is needed to access it are important omissions. It is of some concern that the clinic's specialist psychoanalytical team may remain a hitand-miss opportunity for many people with learning disabilities and, indeed, for the services caring for them.

Unexpected Gains is an interesting and well-written book that can easily be read by (and recommended to) professionals in the field as well as lay people who know or care for someone with a learning disability.
However, I wonder whether it will break into the market (and therefore awareness) outside the confines of the psychotherapeutic community.

Angela Hassiotis Senior Lecturer in Learning Disability, Royal Free and University College Medical School, Wolfson Building, 48 Riding House Street, London WIN 8AA, UK

doi: 10.1192/bjp.189.2.194a

\section{Screening for Perinatal Depression}

Edited by Carol Henshaw \& Sandra Elliott. London: Jessica Kingsley. 2005. 240pp. Ł27.95 (pb). ISBN 1843102196

Making the case for introducing screening for perinatal psychiatric disorders is not without problems. The case is not helped by the common use of 'postnatal depression' as a catch-all term for a variety of conditions, from mild and transient mood lability to some of the most severe psychiatric disorders seen in clinical practice. 'Screening for perinatal depression' means different things to different people and it is appropriate to begin by asking screening by whom, for what and when? To its credit,

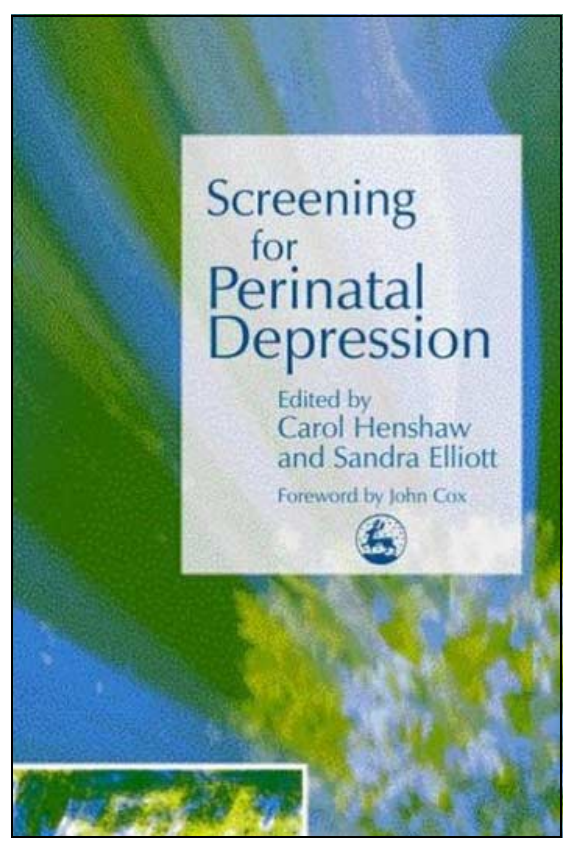

this book considers both antenatal screening for women currently well but at risk of perinatal mood disorder, and screening women for current affective symptoms.

The book, at least in part, can be seen as a response to the controversial decision of the UK National Screening Committee not to recommend the introduction of routine screening for postnatal depression using the Edinburgh Postnatal Depression Scale. A number of different perspectives are brought to the table - a large number of professional groups are represented from both academic and clinical backgrounds. The individual chapters range widely from those describing clinical services, outlining individual research projects or providing personal experiences of accessing services, to those taking a more philosophical overview of the issues involved. In particular the book benefits from a final chapter, written by the editors, responding to many of the issues raised in the previous chapters and summarising the areas of agreement and those that remain in dispute. There is much of interest here to those from a wide variety of professional backgrounds, and the book should have wider appeal than merely perinatal 'anoraks' like me.

One recurring theme is a lack of research evidence - reflected by the fact that a number of chapters rely on a description of local services. It also becomes clear that the title is a misnomer - the important perinatal psychiatric conditions are wider than major depression and, particularly when screening for women at risk, include bipolar and schizoaffective disorders.

A philosophical divide is clear - between those who see postnatal depression as an underdiagnosed illness that, without recognition and treatment, can result in long-term negative consequences for a woman and her family, and those who believe that a diagnosis of postnatal depression is the imposition of an inappropriate medical model on the suffering of women at this time of difficult role transition. In his forword, John Cox expresses the hope that the book will be a 'constructive dialogue' between the different perspectives. Judged on this criterion, I think it can be considered a success.

Ian Jones Neuropsychiatric Genetics Unit, Department of Psychological Medicine, University of Wales College of Medicine, Heath Park, Cardiff CFI4 4XN, UK. Email: jonesirl@cf.ac.uk

doi: 10.II92/bjp.189.2.195 